University of Nebraska - Lincoln

DigitalCommons@University of Nebraska - Lincoln

\title{
Structure and magnetism of atomically thin Fe layers on flat and vicinal Pt surfaces
}

D. Repetto

Max-Planck-Institut für Festkörperforschung

T. Y. Lee

Max-Planck-Institut für Festkörperforschung

S. Rusponi

École Politechnique Fédéral de Lausanne

Jan Honolka

Max-Planck-Institut für Festkörperforschung, honolka@fzu.cz

K. Kuhnke

Max-Planck-Institut für Festkörperforschung

See next page for additional authors

Follow this and additional works at: https://digitalcommons.unl.edu/physicsenders

Part of the Physics Commons

Repetto, D.; Lee, T. Y.; Rusponi, S.; Honolka, Jan; Kuhnke, K.; Sessi, Violetta; Starke, Ulrich; Brune, H.; Gambardella, P.; Carbone, C.; Enders, Axel; and Kern, K., "Structure and magnetism of atomically thin Fe layers on flat and vicinal Pt surfaces" (2006). Axel Enders Publications. 7.

https://digitalcommons.unl.edu/physicsenders/7

This Article is brought to you for free and open access by the Research Papers in Physics and Astronomy at DigitalCommons@University of Nebraska - Lincoln. It has been accepted for inclusion in Axel Enders Publications by an authorized administrator of DigitalCommons@University of Nebraska - Lincoln. 


\section{Authors}

D. Repetto, T. Y. Lee, S. Rusponi, Jan Honolka, K. Kuhnke, Violetta Sessi, Ulrich Starke, H. Brune, P. Gambardella, C. Carbone, Axel Enders, and K. Kern 


\title{
Structure and magnetism of atomically thin Fe layers on flat and vicinal Pt surfaces
}

\author{
D. Repetto, ${ }^{1, *}$ T. Y. Lee, ${ }^{1}$ S. Rusponi,${ }^{2}$ J. Honolka, ${ }^{1}$ K. Kuhnke,,${ }^{1}$ V. Sessi, ${ }^{1}$ U. Starke,${ }^{1}$ H. Brune,${ }^{2}$ \\ P. Gambardella, ${ }^{2,3}$ C. Carbone, ${ }^{4}$ A. Enders, ${ }^{1}$ and K. Kern ${ }^{1,2}$ \\ ${ }^{1}$ Max Planck Institut für Festkörperforschung, Heisenbergstrasse 1, 70569 Stuttgart, Germany \\ ${ }^{2}$ Institut de Physique de Nanostructures, École Politechnique Fédéral de Lausanne, CH-1015 Lausanne, Switzerland \\ ${ }^{3}$ Catalan Institute for Research and Advanced Studies (ICREA) and Catalan Institute of Nanotechnology (ICN), UAB Campus, E-08193 \\ Bellaterra Spain \\ ${ }^{4}$ Istituto della Struttura della Materia C.N.R., Area Science Park, I-34012 Trieste, Italy \\ (Received 26 October 2005; revised manuscript received 27 June 2006; published 4 August 2006)
}

\begin{abstract}
Ultrathin Fe films on Pt substrates have been investigated under ultrahigh vacuum conditions by scanning tunneling microscopy, low energy electron diffraction, magneto-optical Kerr effect, x-ray magnetic circular dichroism measurements, and Kerr microscopy. We present a comparison between Fe films on flat Pt(111) and stepped $\mathrm{Pt}(997)$, with particular focus on the magnetic anisotropy in the submonolayer thickness range below 0.2 monolayer coverage, and above the spin reorientation transition at 3 monolayer thickness. The comparison of structure and magnetism suggests that the perpendicular easy axis found for films thinner than three monolayers is due to dominating contributions from both film interfaces to the anisotropy energy. The Fe-Pt interface contribution has its origin in the hybridization of the $\mathrm{Fe} 3 d$ with the $\mathrm{Pt} 5 d$ band. The in-plane magnetic anisotropy above 3 atomic layers film thickness can be correlated directly with peculiarities of the film structure.
\end{abstract}

DOI: 10.1103/PhysRevB.74.054408

PACS number(s): 75.30.Gw, 75.30.Kz, 75.50.Bb, 75.70.Ak

\section{INTRODUCTION}

Studies addressing the correlation between structure and magnetism of ultrathin films and nanostructures are of undiminished interest, constantly stimulated by the demands of application-oriented research, or in conjunction with advances in the fabrication of complex nanostructures. Epitaxial monolayer thin films and submonolayer structures represent model systems at the crossover between bulk and truly atomic structures and are studied extensively for this reason. ${ }^{1-8}$ To account for the dependence of the magnetism of epitaxial films on electronic and structural film properties, phenomenological quantities, such as magnetocrystalline, magnetoelastic, interface, or step anisotropy energies have been introduced and applied successfully during the past 2 decades of research. ${ }^{3,7,9-13}$ These parameters are chosen to reflect the symmetry of the observed magnetic anisotropy and usually hide details of the sample structure. The immense effort in determining such anisotropy contributions experimentally has partly distracted from gaining a better understanding of the role of the local atomic structure itself. The main reason for this is the limited success in correlating the magnetism to the sometimes minute details in structural properties, which is a result of the complex interplay between local atomic structure, electronic structure, and magnetic anisotropy in thin films. Recently, progress has been made to pinpoint the structural dependence of the magnetism for some systems by studying changes in the magnetism upon structural transitions, either with increasing film thickness or due to thermal activation. We demonstrated, for instance, the close relationship between morphology and domain wall pinning on $\mathrm{Fe} / \mathrm{Cu}(001)$ by monitoring magnetic domain propagation directly during film annealing. ${ }^{14,15}$ Interface contributions, on the other hand, could be determined in gas adsorption experiments, where spin reorientation transi- tions have been stimulated without altering the film structure. ${ }^{16,17}$

In the present work we pursue another approach to reveal structural dependence of the magnetism, and to separate this effect from Neél-type contributions. We exploit differences in the growth mode of $\mathrm{Fe}$ on flat $\mathrm{Pt}(111)$ and stepped $\mathrm{Pt}(997)$ surfaces to fabricate Fe films of different crystalline structure and morphology. The comparison of the magnetism of both systems will allow us to identify the origin of the magnetic anisotropy. We concentrate on (i) the substrate contribution to the magnetic anisotropy, studied for Fe coverages less than 0.2 monolayers (ML) on both substrates, and (ii) the correlation of the in-plane anisotropy with details of the bcc film structure above $3 \mathrm{ML}$ film thickness. The presented results go beyond a recently published detailed study of $\mathrm{Fe}$ on $\mathrm{Pt}(997)$, in which the authors mainly focus on the perpendicular magnetic anisotropy of this system between 0.2 and 3 ML Fe coverage. ${ }^{13}$

\section{EXPERIMENT}

The experiments have been carried out in an ultrahigh vacuum (UHV) chamber system consisting of a preparation and a magnetism chamber, with a base pressure of 8 $\times 10^{-11}$ mbar. The preparation chamber is equipped with standard tools for substrate preparation, film deposition, and structural characterization. The sample temperature can be varied between $110 \mathrm{~K}$ by liquid nitrogen cooling and $1300 \mathrm{~K}$ by electron bombardment heating for the preparation as well as for temperature dependent magnetic characterization. The Pt substrates have been prepared by repeated sputtering/ annealing cycles. The kinetic energy of the $\mathrm{Ar}^{+}$-ions for sputtering is set to $1 \mathrm{keV}$. Sputter damages on the surface are removed by heating the sample up to $870 \mathrm{~K}[\mathrm{Pt}(997)]$ or $1300 \mathrm{~K}[\mathrm{Pt}(111)]$ between the sputtering cycles. From this 
temperature, the sample is slowly cooled down to room temperature $(\mathrm{RT} \sim 298 \mathrm{~K})$ with a rate of $\sim 0.5 \mathrm{~K} / \mathrm{s}$. By this procedure, a regular array of terraces of $20 \AA$ width (8 atomic rows) with (111) orientation separated by monatomic steps (height of $2.26 \AA$ ) is formed with a high degree of ordering. The cleanliness of Pt substrates during and after the preparation has been checked by Auger electron spectroscopy (AES).

Iron is evaporated from a rod with a purity of $99.99 \%$ by electron bombardment. During the film growth, the pressure in the chamber is less than $2 \times 10^{-10}$ mbar. The evaporation rate of the source is typically $0.8 \AA / \mathrm{min}$ as calibrated by depositing on a microbalance prior to each film preparation. The calibration with the microbalance is based on the density of bulk Fe and has to be converted to monolayer (ML) by considering the actual areal density of the adlayers. One pseudomorphic fcc-Fe layer on $\mathrm{Pt}(111)$ corresponds to a thickness of $1.78 \AA$. On the other hand, a bcc-Fe layer corresponds to a thickness of $1.43 \AA\left(\rho_{\mathrm{Pt}}^{f c-111}=1.503\right.$ $\times 10^{15}$ atoms $/ \mathrm{cm}^{2}, \rho_{\mathrm{Fe}}^{b c c-100}=1.214 \times 10^{15}$ atoms $\left./ \mathrm{cm}^{2}\right)$. In the following, the nominal coverage will be given in ML under the assumption of the atomic density of the pseudomorphic fcc-structure for all layers.

Scanning tunneling microscopy (STM) experiments have been performed in a separate chamber on different substrates. Also here, the film and substrate preparation was done following the above-described procedure. Comparative low energy electron diffraction (LEED), AES, and magnetooptical Kerr effect (MOKE) measurements performed on both samples gave identical results.

Magnetic investigations have been performed on films of constant coverage as well as wedge-shaped films with the nominal coverage varying from 0 up to $8.5 \mathrm{ML}$. The height and length of the wedges are determined by the evaporation rate and the speed by which the sample is moved with respect to a metal shutter placed between the sample and the Fe source. The standard wedge length is $4 \mathrm{~mm}$.

For magnetic characterization the sample is transferred in situ into the magnetism chamber. ${ }^{18}$ Here, two electromagnets produce external magnetic fields at the sample position up to $70 \mathrm{mT}$ within the film plane and $40 \mathrm{mT}$ perpendicular to the film plane. The magnetic properties are investigated by integral and laterally resolved MOKE measurements. The former is used to obtain magnetization loops of a sample area of $0.25 \mathrm{~mm}^{2}$. Longitudinal and polar geometries are used for studying the magnetization within the film plane and perpendicular to the film surface. For the MOKE measurements on $\mathrm{Fe} / \mathrm{Pt}(111)$ the angle between the incident laser beam and the surface normal is $\sim 15^{\circ}$. For the experiments on $\mathrm{Fe} / \mathrm{Pt}(997)$, an improved MOKE setup has been used, with the angle of incidence increased to $45^{\circ}$. This modification allows for a good sensitivity for both in-plane and out-ofplane magnetization. We remark that the MOKE signal is not a quantitative measure for the magnetization and depends on the angle of incidence, optical alignment, and other parameters. Furthermore, it is not element-specific and contains integral magnetic information from the film and the substrate. The Kerr microscope permits direct imaging of the magnetization state of the sample within an area of 400 $\times 300 \mu \mathrm{m}^{2}$. A lateral resolution $\leqslant 1 \mu \mathrm{m}$ and optical magni- fication of 10 is achieved. Images are read out by a computer controlled charge coupled device (CCD) camera with an acquisition rate of $0.3 \mathrm{~s} /$ image. The advantage of this setup is that changes in the magnetization state can be observed directly as a function of magnetic field, sample temperature, gas adsorption, etc. For the adsorption experiments, the oxygen has been introduced into the UHV chamber through a leak valve to achieve an oxygen partial pressure of 8.5 $\times 10^{-9}$ mbar.

Due to the limited sensitivity of MOKE we also investigated the magnetism of the very thin Fe films $(<0.5 \mathrm{ML})$ by $\mathrm{X}$-ray magnetic circular dichroism measurements (XMCD) using synchrotron radiation. These experiments allow for quantitative and element specific determination of the film magnetization with high sensitivity and as a function of the angle with respect to the film normal. The XMCD measurements have been performed in the total electron yield mode at the European Synchrotron Radiation Facility (ESRF) in Grenoble/France, at beamline ID-08.

\section{RESULTS}

\section{A. Growth of $\mathrm{Fe}$ on $\mathrm{Pt}$}

The growth of Fe on Pt is currently the subject of extensive research. ${ }^{13,19-22}$ Particular focus has been on the preparation of $\mathrm{Fe}$ on stepped $\mathrm{Pt}(997)$ since it allows one to form linear nanostructures and even chains of single $\mathrm{Fe}$ atoms. Our available data ${ }^{22,46}$ obtained by thermal energy helium atom scattering (TEAS) can be summarized to the following picture: At deposition temperatures between 200 and $400 \mathrm{~K}$, $\mathrm{Fe}$ atoms preferentially decorate the Pt substrate steps at the initial growth stage. ${ }^{13,22}$ With increasing Fe coverage, $\theta_{\mathrm{Fe}}$, the step decoration is continued, resulting in step flow growth mode. It is expected that for $\theta_{\mathrm{Fe}}>1 \mathrm{ML}$, the film overgrows the substrate steps, leading to vertical mismatch and increasing disorder. The step periodicity is thus progressively lost.

Within the work presented here, the growth of Fe on flat $\mathrm{Pt}(111)$ has been investigated by STM and LEED, for comparison. In Fig. 1 STM images of different $\theta_{\mathrm{Fe}}$ on $\operatorname{Pt}(111)$ are shown. On the flat Pt surface the formation of a 3D morphology is observed already at the initial stage of the growth. The $3 \mathrm{D}$ islands have irregular, ramified shape and grow laterally and in height with increasing coverage. A nominal $\theta_{\mathrm{Fe}}$ of 1.7 ML is distributed into four simultaneously open layers. The corrugations visible in the second and third layer in the contrast-enhanced image in Fig. 1(d) are a network of misfit dislocations removing part of the stress resulting from the lattice mismatch between $\mathrm{Fe}$ and $\mathrm{Pt}^{23,44}$ The first monolayer is pseudomorphic since it has a uniform apparent height and therefore no surface partial dislocations. Significant diffusion of $\mathrm{Fe}$ into the Pt sets in only above $600 \mathrm{~K} .{ }^{20,22} \mathrm{We}$ remark that there is a weak tendency for $\mathrm{Fe}$ incorporation into the $\mathrm{Pt}$ top layer at very low dilution already around $140 \mathrm{~K}$, which may be ascribed to the relaxation of the compressive stress in the clean $\operatorname{Pt}(111)$ surface. ${ }^{24}$

LEED investigations reveal complementary information on the atomic film structure. Figure 2(a) displays a LEED 

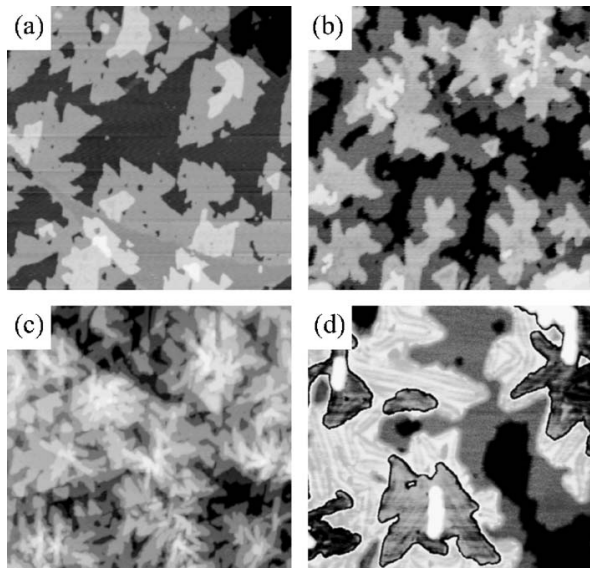

FIG. 1. (a) and (b) STM on 0.7 ML Fe, 1.25 ML Fe, image size $150 \times 150 \mathrm{~nm}^{2}$. (c) $2.5 \mathrm{ML} \mathrm{Fe}$ on $\mathrm{Pt}(111)$, image size 150 $\times 138 \mathrm{~nm}^{2}$. (d) $1.7 \mathrm{ML} \mathrm{Fe} / \mathrm{Pt}(111)$, image size $60 \times 55 \mathrm{~nm}^{2}$. In (d) the contrast was adjusted to emphasize the corrugation in the second layer. $T_{\text {growth }}=300 \mathrm{~K}$.

image taken on $0.8 \mathrm{ML} \mathrm{Fe} / \mathrm{Pt}(111)$. The six diffraction spots of the fcc-(111) surface are slightly elongated in the radial direction. The second-order diffraction spots, not visible at this energy, show an elongation in tangential direction. The symmetry of the diffraction spots hence excludes the presence of a pure fcc-Fe layer. A comparison with the STM images (Fig. 1) shows that at this coverage of $0.8 \mathrm{ML}$ also the second and even the third layer contribute to the diffraction image. The observation of dislocation lines in the second layer in the STM images and the anisotropic spot broadening in the LEED pattern is thus interpreted as a structural transformation from fcc to bcc from the second Fe layer on.

By increasing $\theta_{\mathrm{Fe}}$ further, the LEED pattern visibly changes. For $3 \mathrm{ML} \mathrm{Fe} / \mathrm{Pt}(111)$, around each first-order diffraction spot, six characteristic satellite spots can be observed [Fig. 2(b)]. The intensity of the satellite spots depends strongly on the kinetic energy of the primary electrons due to the limited sensitivity of LEED to the structure along the surface normal. At no energy all satellite spots could be seen with identical intensity. ${ }^{25}$ To obtain this image, the sample was annealed at $500 \mathrm{~K}$ to improve the quality of the satellite spots which are already visible before annealing. The arrangement of these spots is consistent with the assumption of a bcc-Fe layer on $\mathrm{Pt}(111)$, as will be explained in Sec. IV. a)

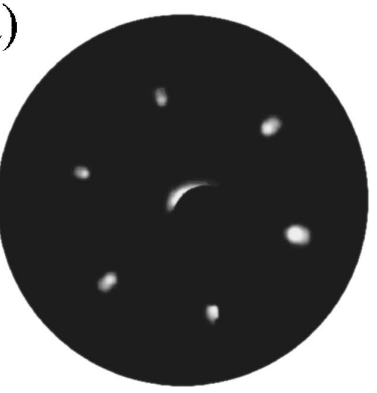

b)

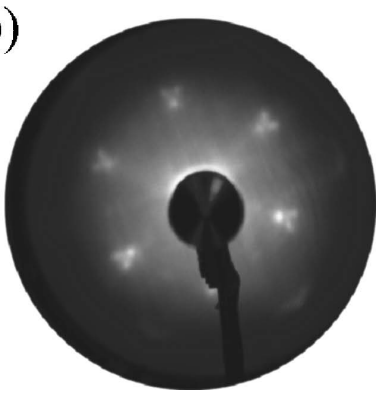

FIG. 2. LEED images taken at $152 \mathrm{eV}$ on $\mathrm{Fe} / \mathrm{Pt}(111)$ grown at $300 \mathrm{~K}$ : (a) $\Theta_{\mathrm{Fe}}=0.8 \mathrm{ML}$ and (b) $\Theta_{\mathrm{Fe}}=3 \mathrm{ML}$ after annealing at $500 \mathrm{~K}$.
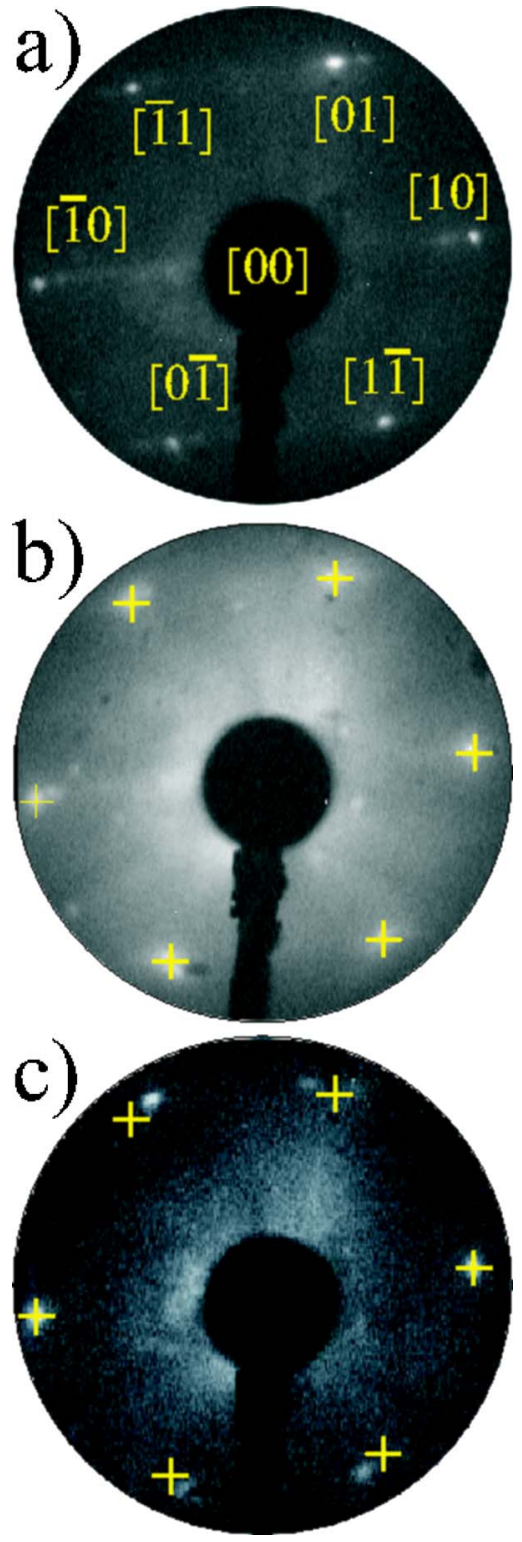

FIG. 3. (Color online) LEED images taken at $122 \mathrm{eV}$ on $\mathrm{Fe} / \mathrm{Pt}(997)$ grown at $300 \mathrm{~K}$, (a) $\Theta_{\mathrm{Fe}}=1 \mathrm{ML}$, (b) $\Theta_{\mathrm{Fe}}=2.2 \mathrm{ML}$, and (c) $\Theta_{\mathrm{Fe}}=6.6 \mathrm{ML}$. Crosses ( + ) mark the position of the substrate fcc(111) diffraction spots.

Complementary LEED images obtained on $\mathrm{Fe} / \mathrm{Pt}(997)$ are shown in Figs. 3(a)-3(c). In Fig. 3(a) a characteristic diffraction image of $1 \mathrm{ML} \mathrm{Fe} / \mathrm{Pt}(997)$ is shown. This image corresponds to the LEED pattern of the clean Pt(997) substrate without qualitative difference. The superstructure resulting from the substrate steps is fully maintained. We conclude that a pseudomorphic Fe layer has been formed. From a nominal coverage $\theta_{\mathrm{Fe}}$ of $1 \mathrm{ML}$ on, the superstructure spots merge into broad spots with hexagonal symmetry [Fig. 3(b)]. In addition, the background intensity increases. The observed hexagonal pattern indicates that Fe still grows with fccstructure in the second and third layers in registry with the substrate. A characteristic change of the diffraction image is observed at $\theta_{\mathrm{Fe}} \simeq 3 \mathrm{ML}$ : four $[(01),(1 \overline{1}),(0 \overline{1}),(\overline{1} 1)]$ of the 


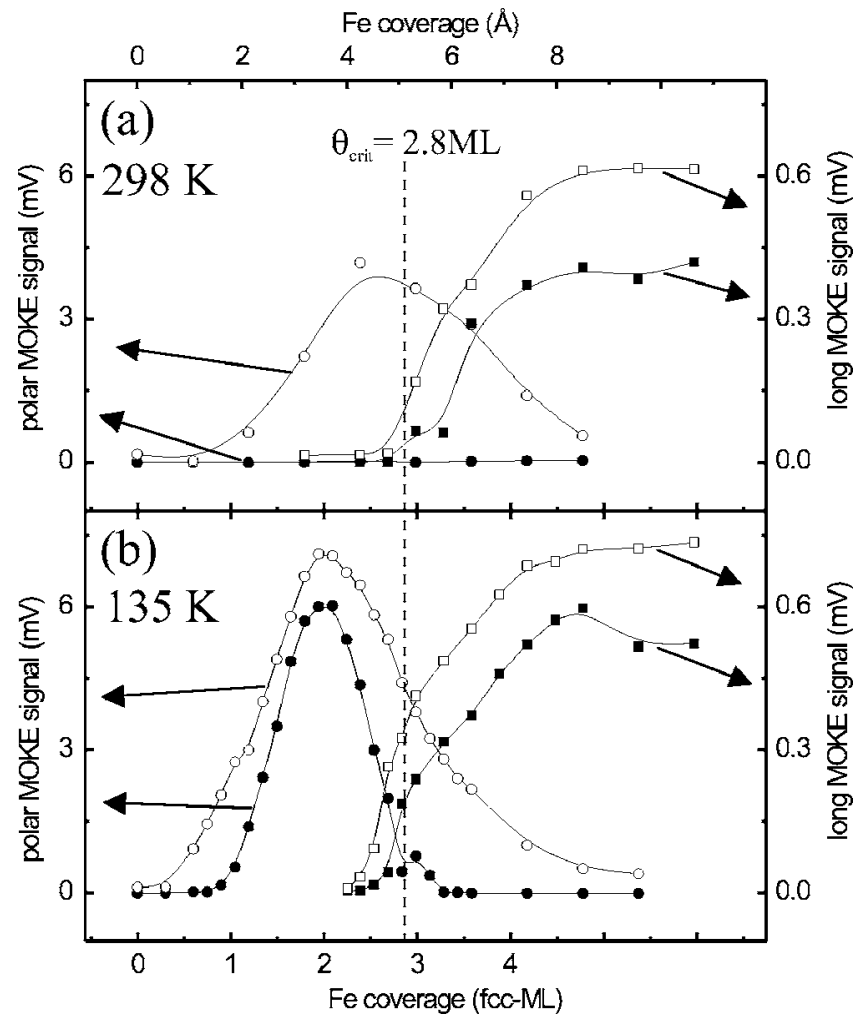

FIG. 4. Polar remanent $(\bullet)$, polar saturation $(\bigcirc)$, longitudinal remanent ( $\square$ ), and longitudinal saturation $(\square)$ Kerr intensities of MOKE hysteresis loops taken as a function of Fe coverage on a Fe-wedge on $\mathrm{Pt}(111)$ at $298 \mathrm{~K}$ (a) and $135 \mathrm{~K}$ (b). The Fe thickness increases linearly from 0 to $4.8 \mathrm{ML}$ and then remains constant.

six first-order diffraction spots shift with respect to the fccposition, while the (10) and (10) spots stay fixed at the same position [Fig. 3(c)]. To visualize the change, the substrate first-order spot positions are marked in the images $(+)$. The observed LEED images will be interpreted as a fcc-bcc structural transition in the following section.

\section{B. Magnetism of $\mathrm{Fe}$ on $\operatorname{Pt}(111)$ and $\operatorname{Pt}(997)$}

The magnetism of $\mathrm{Fe}$ on $\mathrm{Pt}(111)$ was investigated on wedge-shaped films by magneto-optical Kerr effect measurements as a function of Fe coverage $\theta_{\mathrm{Fe}}$. The evolution of the magnetization with $\theta_{\mathrm{Fe}}$ is shown in Fig. 4 for two different measurement temperatures, $298 \mathrm{~K}$ (a) and $135 \mathrm{~K}$ (b). The remanent and saturation Kerr signals have been derived from MOKE hysteresis loops (Fig. 5). Clearly visible is a spin reorientation transition (SRT) from in-plane to out-of-plane when $\theta_{\mathrm{Fe}}$ is reduced below a critical coverage of $\theta_{\text {crit }} \sim 2.8$ ML. Such a SRT is found for many thin-film/substrate systems and is commonly ascribed to the dominant role of the interface anisotropy. The polar magnetization shows a strong temperature dependence in the investigated temperature range. While at RT no remanent Kerr signal was observed [Fig. 4(a)], stable ferromagnetic order was found if the sample was cooled below $150 \mathrm{~K}$ [Fig. 4(b)]. For submonolayer coverages below $\theta_{\mathrm{Fe}}=0.85 \mathrm{ML}$ no remanent magnetization could be obtained in the MOKE experiments at
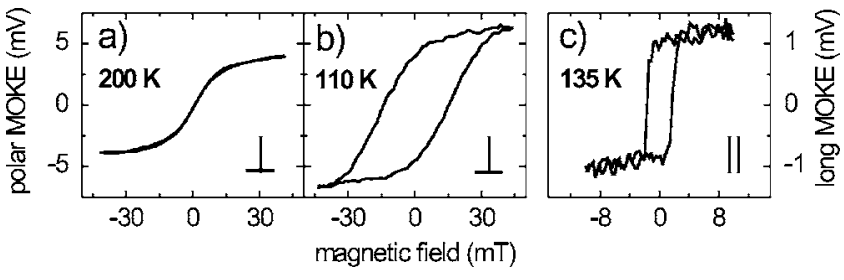

FIG. 5. Polar MOKE hysteresis loops of $1.4 \mathrm{ML} \mathrm{Fe/Pt(111)} \mathrm{at}$ $200 \mathrm{~K}$ (a) and $110 \mathrm{~K}$ (b); and longitudinal MOKE loop of 4.5 ML $\mathrm{Fe} / \mathrm{Pt}(111)$ at $110 \mathrm{~K}(\mathrm{c})$.

$110 \mathrm{~K}$. This result is consistent with the assumption that hysteresis appears when the film has passed the percolation threshold. For a film growing from monolayer islands this value is typically $0.65 \mathrm{ML}{ }^{26}$ In the present case, part of the deposited $\mathrm{Fe}$ is present in the second layer pushing the percolation and therefore the onset of hysteresis to higher coverages. The longitudinal remanent and saturation Kerr intensities ( $\boldsymbol{\square}$ and $\square$, respectively) increase with Fe thickness and remain constant on the plateau of the wedges. There is no sign of a magnetic anisotropy within the film plane. The MOKE loops measured with the field applied along the [ $\overline{1} 10]$ and $[11 \overline{2}]$ directions are both square-shaped and appear to be identical. Within a limited coverage range around $\theta_{\text {crit }}$ polar and longitudinal Kerr signals coexist.

Comparative magnetic investigations have been done on similar Fe films of varying thickness (0-6.7 ML) prepared on stepped $\operatorname{Pt}(997)$ substrates. The results of the thickness dependent MOKE measurements on these films at $300 \mathrm{~K}$ are summarized in Fig. 6. As for Fe/Pt(111), also on the Pt(997) substrates a SRT from in-plane to perpendicular magnetization is observed. The $\theta_{\text {crit }} \sim 3.3 \mathrm{ML}$ is $\sim 15 \%$ larger than for $\mathrm{Fe} / \mathrm{Pt}(111)$. No magnetic signal was measured below $\theta_{\mathrm{Fe}}$ $\sim 1 \mathrm{ML}$ at this temperature. For this thickness range, ferromagnetic hysteresis loops can again be obtained by cooling the sample below $200 \mathrm{~K}$. Note that the found $\theta_{\text {crit }}$ is almost

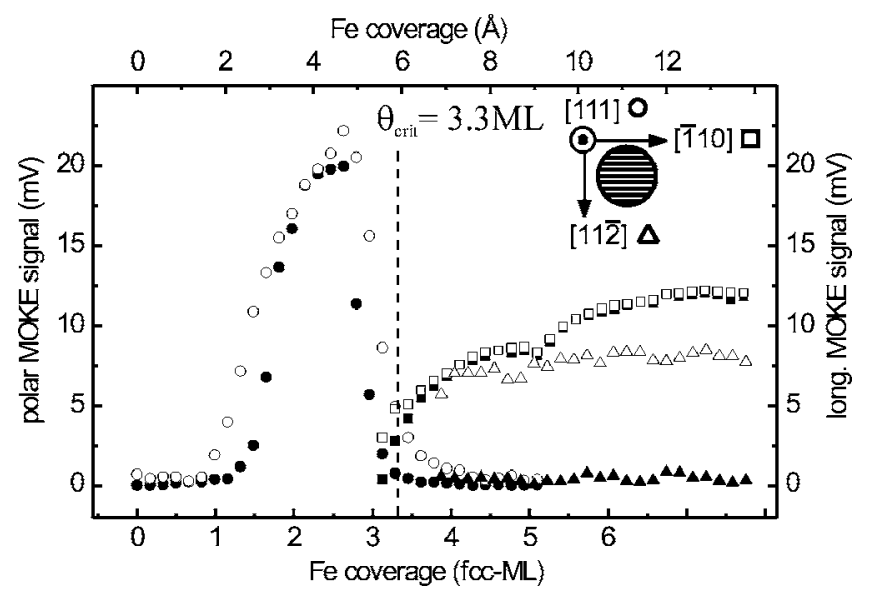

FIG. 6. Kerr intensities of MOKE hysteresis loops taken at $300 \mathrm{~K}$ on an Fe-wedge $/ \mathrm{Pt}(997)$ as a function of $\theta_{\mathrm{Fe}}$ : polar remanence $(\bigcirc)$, polar saturation $(\bigcirc)$, longitudinal remanence along [1110] (ם), longitudinal saturation along $[\overline{1} 10](\square)$, longitudinal remanence along $[11 \overline{2}](\mathbf{\Delta})$, and longitudinal saturation along [11 $\overline{2}]$ $(\triangle)$. 

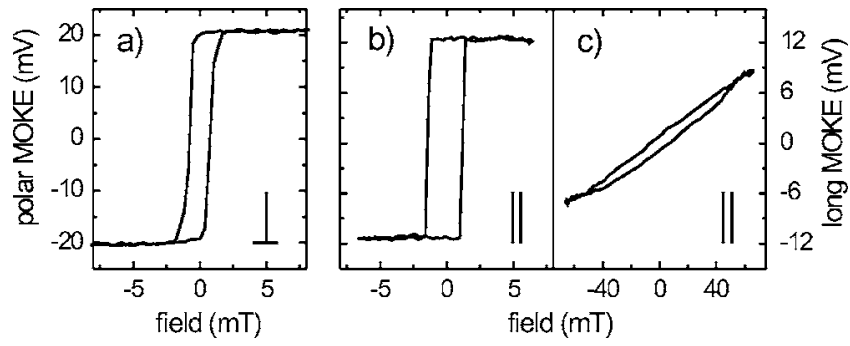

FIG. 7. Polar $(\perp)$ and longitudinal $(\|)$ MOKE hysteresis loops taken at $300 \mathrm{~K}$ on (a) $2.5 \mathrm{ML} \mathrm{Fe} / \mathrm{Pt}(997)$, (b) $6.5 \mathrm{ML} \mathrm{Fe/Pt(997)}$ along the $[\overline{1} 10]$ direction parallel to the substrate steps, and (c) 6.5 $\mathrm{ML} \mathrm{Fe} / \mathrm{Pt}(997)$ along [112] perpendicular to the steps.

twice as large as the value reported in Ref. 13 .

The longitudinal MOKE measurements have been done by applying the magnetic field within the film plane parallel

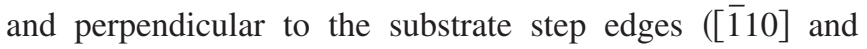
[112]) on separate films, after rotating the substrate by $90^{\circ}$. The measurements show a striking directional dependence of the magnetization within the film plane above $\theta_{\text {crit }}$. Along the step edges the saturation and the remanent Kerr signals have similar values. In contrast, no remanence was observed perpendicular to the step edges $(\boldsymbol{\Delta})$. The signal represented by $(\triangle)$ corresponds to the Kerr signal at maximum available field and does not correspond to full magnetic saturation.

Characteristic MOKE magnetization loops taken on $\mathrm{Fe} / \mathrm{Pt}(997)$ below and above $\theta_{\text {crit }}$ are shown in Fig. 7. The polar MOKE loop taken at $\theta_{\mathrm{Fe}}=2.5 \mathrm{ML}$ in (a) is clearly square-shaped at RT, in contrast to the loops obtained for the same $\theta_{\mathrm{Fe}}$ on $\mathrm{Pt}(111)$ at this temperature (Fig. 5). The in-plane loops taken at $\theta_{\mathrm{Fe}}=6.5 \mathrm{ML}$ along and perpendicular to the substrate steps (b) and (c) reflect the pronounced in-plane anisotropy with the easy axis along the step edges.

We performed complementary $\mathrm{x}$-ray magnetic circular dichroism (XMCD) measurements for submonolayer Fe coverages on flat and stepped Pt substrates. The angular dependence of the normalized dichroic signal of submonolayer Fe coverages on $\operatorname{Pt}(997)(0)$ and $\operatorname{Pt}(111)(\square)$ is shown in Fig. 8. The plotted quantity, XMCD/XAS, is to first order proportional to the total magnetic moment per Fe atom, projected on the wave vector of the X-ray beam. On the $\operatorname{Pt}(997)$ only a small amount of $0.13 \mathrm{ML} \mathrm{Fe}$ has been deposited at $300 \mathrm{~K}$. Due to the surface mobility of the impinging Fe atoms at this temperature monatomic Fe chains are formed by substrate step decoration. In contrast, 0.31 ML Fe have been deposited on the $\mathrm{Pt}(111)$ at $10 \mathrm{~K}$ to suppress surface diffusion. As a result, very small ramified $\mathrm{Fe}$ islands with an average number of Fe nearest neighbors per Fe atom of $N N_{\mathrm{Fe}-\mathrm{Fe}}=2.2$ are obtained. ${ }^{45}$ Thus the $N N_{\mathrm{Fe}-\mathrm{Fe}}$ is comparable for both samples while number of Pt nearest neighbors per Fe, $N N_{\mathrm{Fe}-\mathrm{Pt}}$, is different. The measurements in Fig. 8 show that the preferential magnetization direction for $\mathrm{Fe} / \mathrm{Pt}(111)$ is in the polar direction also for very small Fe coverage. In contrast, the angular measurements for monatomic chains on $\operatorname{Pt}(997)$ reveal that the easy magnetization axis is close to the film plane, but perpendicular to the chain axis. A small canting angle of the magnetization vector of $10^{\circ}$ with respect to the surface plane

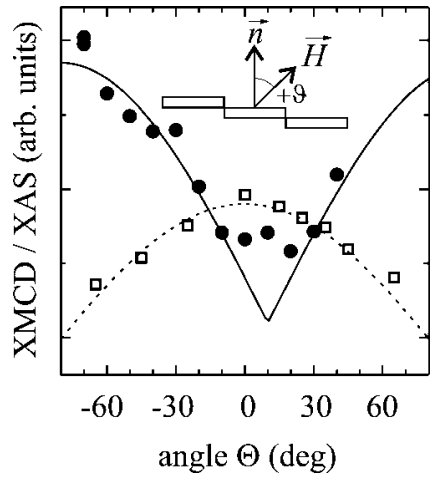

FIG. 8. Angular dependence of the normalized XMCD signal at the $L_{3}$ edge of monatomic chains [0.13 ML Fe/Pt(997), O and small $\mathrm{Fe}$ islands $[0.31 \mathrm{ML} \mathrm{Fe} / \mathrm{Pt}(111), \square]$, measured in external field of $1 \mathrm{~T}$ at $6 \mathrm{~K}$. The angle $\vartheta$ is measured with respect to the surface normal, in the plane perpendicular to the substrate step edges. The dashed and continuous lines are guides to the eye, based on a $\left|\cos \left(\vartheta-\vartheta_{0}\right)\right|$ function, with $\vartheta_{0}=0^{\circ}$ and $10^{\circ}$ for $\operatorname{Pt}(111)$ and $\mathrm{Pt}(997)$, respectively.

is found. It can be concluded from the XMCD measurements that the polar magnetization in $\mathrm{Fe} / \mathrm{Pt}(111)$ persists also in the submonolayer coverage range down to the limit of single atom impurities, while for $\mathrm{Fe} / \mathrm{Pt}(997)$ a second spin reorientation transition into the plane is found for coverages below $0.5 \mathrm{ML} \mathrm{Fe}$.

\section{Adsorbate effects on the Fe film magnetism}

Oxygen adsorption experiments have been performed on $2 \mathrm{ML} \mathrm{Fe} / \mathrm{Pt}(997)$ to probe the sensitivity of the film magnetism to modification of the electronic structure at the Fevacuum interface. Polar MOKE loops were recorded as a function of exposure to oxygen [Fig. 9(a)]. The square shaped magnetization loop of the as-grown film (I) changes to an S-shaped loop with no remanence, but almost unmodified saturation (II). The saturation disappears for dosages higher than $8 \mathrm{~L}$ of $\mathrm{O}_{2}\left(1 \mathrm{~L}=1 \times 10^{-6}\right.$ Torr s) (III), but can be recovered by cooling the sample to $110 \mathrm{~K}$ (IV). No longitudinal MOKE signal was observed during the adsorption process.

In Fig. 9(b), the evolution of the remanent (O) and saturation $(\bigcirc)$ Kerr signals with oxygen exposure, as obtained from polar MOKE hysteresis loops, is shown. For small amounts of $\mathrm{O}_{2}$, less than $1.5 \mathrm{~L}$, a drop of the remanence is observed while the saturation remains constant. Further adsorption of oxygen causes a decreasing also of the saturation signal. Above $10 \mathrm{~L}$, neither polar nor longitudinal MOKE signals have been detected at RT. By lowering the temperature to $110 \mathrm{~K}$, the polar remanent and saturation MOKE signals could again be recovered.

Oxygen induced modifications of the Fe magnetization can be monitored directly during adsorption by in situ Kerr microscopy. The Kerr images in Fig. 10 have been taken during oxygen adsorption at RT on 1.6 ML Fe/Pt(997). The sample has been magnetized first by an external field to obtain a remanent magnetic single domain state. Exposure to 
a)
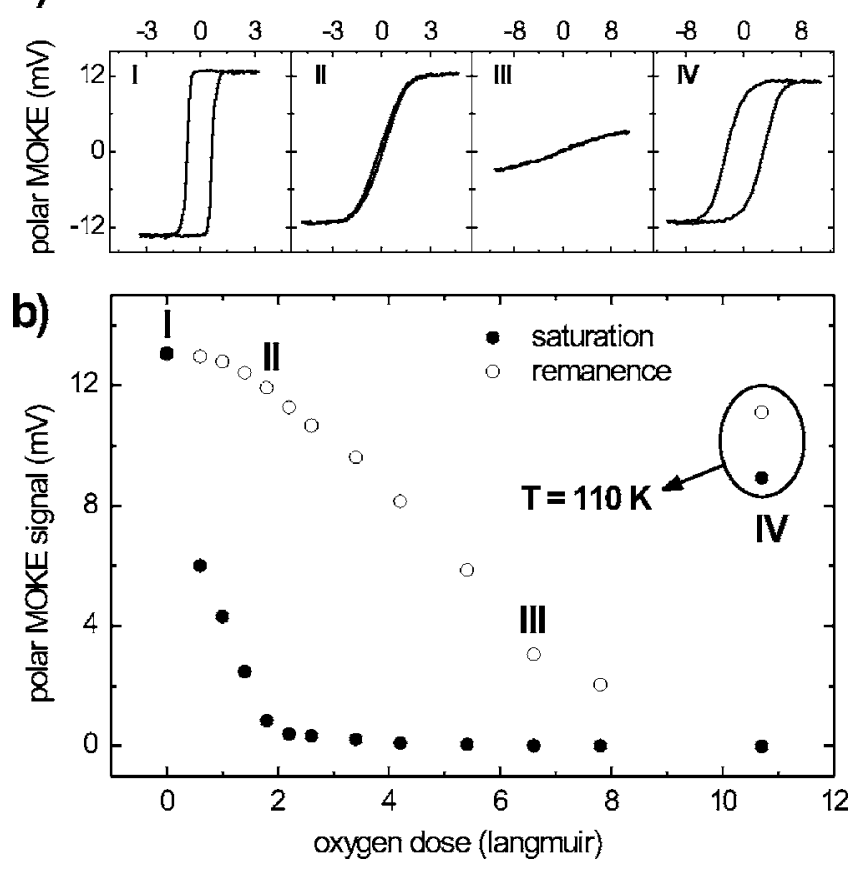

FIG. 9. (a) Polar MOKE hysteresis loops of $2 \mathrm{ML} \mathrm{Fe/Pt(997)}$ taken at RT (I-III) and $110 \mathrm{~K}$ (IV): (I) directly after the Fe growth, (II) after the adsorption of $1.8 \mathrm{~L}$, (III) $6.6 \mathrm{~L}$, and (IV) $10.7 \mathrm{~L}$ of $\mathrm{O}_{2}$ at RT. (b) Remanent $(\bigcirc)$ and saturation $(\bigcirc)$ Kerr intensity of polar MOKE measurements taken during oxygen adsorption at RT as a function of the oxygen dosage.

more than $0.35 \mathrm{~L}$ of $\mathrm{O}_{2}$ causes the appearance of bright stripes which grow mainly in the $[\overline{1} 10]$ direction with increasing $\mathrm{O}_{2}$ exposure. The stripe width of $\sim 20 \mu \mathrm{m}$ is much larger than the terrace width $(\sim 20 \AA)$. After the adsorption

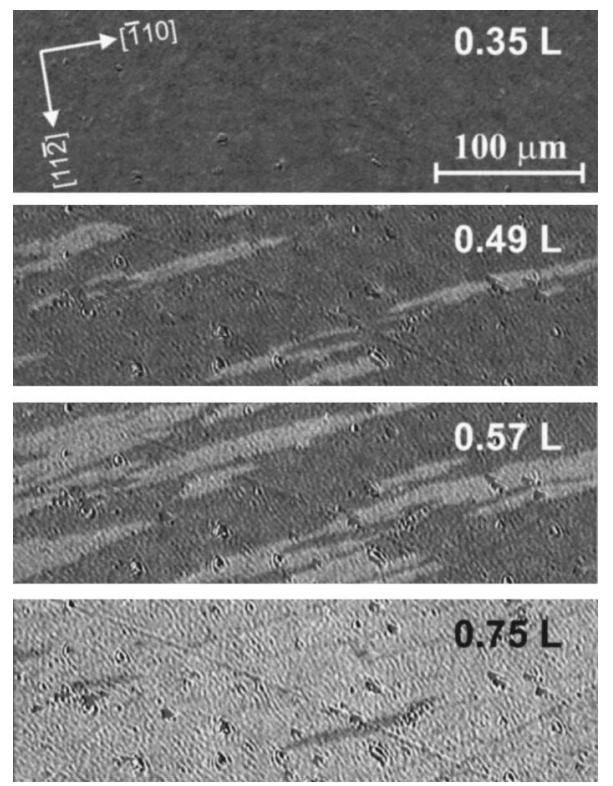

FIG. 10. Kerr microscope images of 1.6 ML Fe/Pt(997) taken during oxygen adsorption at RT as a function of oxygen dosage. Image size: $400 \times 125 \mu \mathrm{m}^{2}$. of $0.75 \mathrm{~L}$ all magnetic contrast has disappeared. The intermediate gray color of the Kerr image after oxygen exposure does not correspond to perpendicular magnetization and may be attributed to a state with no preferential direction for the magnetization, corresponding to a paramagnetic state. However, the strong polarization response in an applied field [see Fig. 9(a) II] rather indicates a transition from a single to a multidomain state with domains too small to be resolved by a Kerr microscope, i.e., smaller than $1 \mu \mathrm{m}$.

\section{DISCUSSION}

The comparative structural and magnetic characterization of ultrathin Fe films on Pt substrates with and without substrate steps reveal striking structural as well as magnetic transitions as a function of $\theta_{\mathrm{Fe}}$. Fe films on $\mathrm{Pt}(111)$ as well as on $\operatorname{Pt}(997)$ show a spin reorientation transition (SRT) from perpendicular to in-plane at a similar critical coverage, $\theta_{\text {crit }}$, of 2.8 and 3.3 ML, respectively. Only for $\mathrm{Fe} / \mathrm{Pt}(997)$ a second SRT is found in the submonolayer regime below 0.5 ML. The structural analysis showed that $\mathrm{Fe}$ starts to grow pseudomorphically on both substrates. The resulting tremendous elastic energy triggers a structural transition towards bcc(110) already in the second atomic layer of $\mathrm{Fe} / \mathrm{Pt}(111)$, which is forming already at a nominal coverage as low as 0.3 ML. The onset of the fcc-bcc transition is delayed in the presence of substrate steps and found at $\sim 3.2 \mathrm{ML}$ for $\mathrm{Fe} / \mathrm{Pt}(997)$. Because of the coincidence of the SRT with the fcc-bcc transition in $\mathrm{Fe} / \mathrm{Pt}(997)$ it is tempting to ascribe the perpendicular anisotropy to the fcc-phase of $\mathrm{Fe}$. In this picture, a transition towards bcc would change the Neél-type surface anisotropy and trigger the SRT. However, the fact that $\mathrm{Fe}$ on $\mathrm{Pt}(111)$ has a very similar $\theta_{\text {crit }}$, despite its different crystalline structure and morphology, opposes such a simple correlation. In the following we will have a closer look on the dependence of the magnetic anisotropy on interface effects on one hand, and on the crystalline structure on the other.

We start with discussing film-substrate interface effects first, on the basis of the data shown in Fig. 8. Fe-Pt coordination effects have been studied on low-dimensional $\mathrm{Fe}$ structures. If the Fe film on $\mathrm{Pt}(997)$ is reduced to the atoms at the substrate steps only, the symmetry of the coordinating Pt atoms determines the direction of the magnetization. The easy axis within the plane and perpendicular to the wire axis is a result of the Fe-Pt interaction, since for free-standing $\mathrm{Fe}$ wires a preferred magnetization axis along the wire axis is expected. ${ }^{27}$ Without the Pt step edge atoms, each $\mathrm{Fe}$ atom is located in a hollow site at the surface and threefold coordinated to Pt. The easy axis is along the surface normal in this case, as has been shown. Of importance for the interaction at the Fe-Pt interface is the hybridization between $\mathrm{Fe} 3 d$ and Pt $5 d$ states, as has been discussed by several authors for various Fe-Pt systems. ${ }^{28-30,47}$ Our element specific XMCD measurements indeed reveal a dichroic signal at the Pt $N_{7,6}$ edge in FePt surface monolayers and will be published elsewhere. The $\mathrm{Fe}$ polarizes the surrounding $\mathrm{Pt}$ atoms which then, owing to their large spin-orbit interaction, significantly contribute to the magnetocrystalline anisotropy (MAE) of the 
whole film-substrate system. In other words, the $\mathrm{Fe}$ is the source of magnetism while the strength of the MAE and the easy magnetization direction is dictated by the polarized $\mathrm{Pt}^{29}$ This contribution to the MAE is by far larger than other contributions, such as the shape anisotropy, and thus dominate the overall magnetic behavior. The differences in the magnetism of $\mathrm{Fe}$ chains and small $\mathrm{Fe}$ islands, which have comparable $N N_{\mathrm{Fe}-\mathrm{Fe}}$ but different $N N_{\mathrm{Fe}-\mathrm{Pt}}$, is a good demonstration of the film-substrate interaction. Another signature of the Pt contribution to the MAE is the large coercivity of $\mathrm{Fe} / \mathrm{Pt},{ }^{13}$ in comparison, for instance, to $\mathrm{Fe} / \mathrm{Cu} .{ }^{14}$

On the other hand, also the film-vacuum interface is of relevance for the perpendicular anisotropy in the present system. The transition to S-shaped magnetization loops in Fig. 9(a) is ascribed to an oxygen-induced decrease of the anisotropy energy contribution arising at the film-vacuum interface, $K_{f-v a c}$. Unlike in other systems studied so far ${ }^{16,17,31}$ oxygen does not cause a spin reorientation into the film plane here. The decrease of $K_{f-v a c}$ might rather result in the formation of magnetic domains which are too small to be resolvable in the Kerr image. Such a multidomain state decreases the magnetostatic energy of the film significantly and becomes energetically favorable if the anisotropy energy or the exchange energy become sufficiently small. Cooling to $110 \mathrm{~K}$ after oxygen adsorption would increase the anisotropy again and help to recover the single domain state. Such a transition from single domain state to a multi- or striped domain state near the SRT is well-established experimentally and theoretically for several systems. ${ }^{32-34}$

We believe that the interaction between $\mathrm{Fe}$ and oxygen is the reason for the reduced surface anisotropy. This conclusion is founded on the experimental observation of strong electronic interaction between Fe and oxygen, with consequences for the Fe's magnetic properties. It was found that the density of states and the spin polarization of Fe near $E_{F}$ decrease gradually with oxygen coverage. ${ }^{35} \mathrm{~A}$ charge transfer from iron to oxygen was furthermore suggested, resulting in spin-polarized states also in the oxygen. Similar effects were observed also for Fe films on different substrates..$^{35,36} \mathrm{We}$ conclude that the anisotropy contributions from the filmsubstrate and the film-vacuum interfaces, $K_{f-s}$ and $K_{f-v a c}$, are of positive sign, meaning that they both promote perpendicular magnetization. After oxygen adsorption, the total surface anisotropy of the film, $K_{S}=\left(K_{f-v a c}+K_{f-s}\right)$, decreases due to the reduced $K_{f-v a c}$, but remains positive since $K_{f-s}$ is unaffected. We would like to point out that oxygen-induced changes of the exchange interaction can have similar consequences for the magnetism as observed in our experiments, but cannot separately be determined with the available data.

In the following, we would like to correlate structural aspects, as presented in Sec. III A, with the magnetism of Fe films thicker than 3.3 ML on Pt(997), described in Sec. III B. The structural transition towards bcc occurs by formation of bcc(110) domains in the so-called Kurdjumov-Sachs (KS) orientation to the underlying fcc(111) layer. Such a structural orientation has been shown previously for $\mathrm{Fe}$ films on $\mathrm{Cu}(111) .{ }^{37}$ In the $\mathrm{KS}$ orientation, the bcc[111] direction is coincident with the fcc[1 $\overline{1} 10]$ direction, bringing two atoms of the sketched bcc(110) cell out of registry as is shown in Fig.
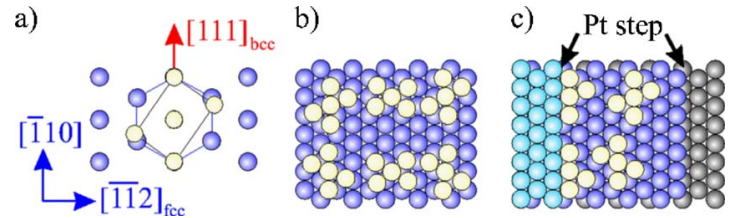

FIG. 11. (Color online) (a) bcc-Fe domains with KurdjumovSachs orientation. Yellow (bright) and blue (dark) spheres represent $\mathrm{Fe}$ and Pt atoms, respectively. The [111] direction of the bccdomains in the adlayer coincides with the $[\overline{1} 10]$ fcc direction of the substrate. For simplicity, the Fe atoms are placed in on-top sites. (b) Atomistic picture of the bcc-Fe domains with Kurdjumov-Sachs orientation on the $\mathrm{Pt}(111)$ substrate, with the Fe atoms in the correct hollow sites. (c) In the presence of substrate steps only 2 bcc KS domains-for better recognition placed on the substrate terrace-remain.

11(a). On the fcc(111) surface six structural domains can be formed [Fig. 11(b)], resulting in satellite diffraction spots in the LEED image in Fig. 2(b). From these satellite spots only one remains visible at the $(01),(1 \overline{1}),(0 \overline{1})$, and ( $\overline{1} 1)$ firstorder diffraction spots for Fe coverage larger than $3 \mathrm{ML}$ on $\mathrm{Pt}(997)$ [Fig. 3(c)]. No satellite spots appear at the (10) and (10) spots. The LEED image is consistent with the hard sphere model given in Fig. 11(c). The formation of four out of six possible bcc-domains is suppressed, and only those domains with the bcc[111] direction aligned along the substrate steps are present. Such a film structure may be the result of almost unhindered strain relaxation perpendicular to the step direction allowing for an incommensurate structure in this direction and will lead to anisotropic stress. The suggested model implies that the substrate steps still influence the film growth above $3 \mathrm{ML}$, as they are the driving mechanism for the nucleation process in the third layer. This bcc layer with its two structural domains, of course, is the template for the nucleation of successive layers with the same structure.

The structural asymmetry is directly reflected in the magnetism of the Fe films above 3 ML thickness. The presence of the Pt(997) substrate steps results with a pronounced inplane anisotropy of the magnetization with the easy axis along the step direction and the hard axis perpendicular to it [Figs. 7(b) and 7(c)]. The effect of the steps is twofold here: on one hand they interrupt the Fe layer, and the modified coordination at the edges gives rise to Neél-type step anisotropy ${ }^{38}$ as found for many other systems. ${ }^{39-41}$ On the other hand, the presence of the step edges directly alters the crystalline structure of the film, which further contributes to the surface anisotropy. It is interesting to compare this system with Fe films grown on other substrates. Fe on vicinal $\mathrm{Cu}(111)$, for instance, also shows in-plane anisotropy, but with all possible six Kurdjumov-Sachs domains present. ${ }^{42}$ The authors could prove an orbital moment anisotropy within the plane due to broken bonds at the steps. This also results in a preferred magnetization direction within the plane and perpendicular to the substrate steps, just as described for the $\mathrm{Fe} / \mathrm{Pt}$ system in this paper, but which is independent of the bcc structure of the film. 


\section{CONCLUSION}

We have shown that ultrathin Fe films exhibit significant differences in their crystallographic structure and morphology when grown on flat $\mathrm{Pt}(111)$ substrates on one hand, and on stepped $\mathrm{Pt}(997)$ on the other. We exploited these differences to study the origin of the magnetic anisotropy in these systems. The comparison of the magnetism of monatomic Fe chains with small islands of similar Fe-Fe coordination but different Fe-Pt coordination reveals the importance of $\mathrm{Fe}-\mathrm{Pt}$ hybridization at the film-substrate interface for the anisotropy of nanostructures and thin films. Oxygen adsorption experiments, on the other hand, evidenced the film-vacuum interface contribution. The crystalline film structure itself seems to be only of minor importance for the polar magnetization. In contrast to that, we show a direct correlation between atomic structure and in-plane anisotropy for the bccfilms at higher Fe coverage.
*Electronic address: diego.repetto@uv.es; URL: http://www.mpistuttgart.mpg.de/kern

${ }^{1}$ U. Gradmann, J. Magn. Magn. Mater. 100, 481 (1991).

${ }^{2}$ J. Thomassen, B. Feldmann, and M. Wuttig, Surf. Sci. 264, 406 (1992).

${ }^{3}$ U. Gradmann, in Handbook of Magnetic Materials, edited by K. H. J. Buschow (Elsevier, Amsterdam, 1993), Vol. VII.

${ }^{4}$ R. Allenspach, J. Magn. Magn. Mater. 129, 160 (1994).

${ }^{5}$ B. Heinrich, in Ultrathin Magnetic Structures, edited by B. Heinrich and J. A. C. Bland (Springer-Verlag, Berlin, 1994), Vol. II, and references therein.

${ }^{6}$ J. Shen and J. Kirschner, Surf. Sci. 500, 300 (2002).

${ }^{7}$ F. Bisio, R. Moroni, F. Bautier de Mongeot, M. Canepa, and L. Mattera, Phys. Rev. Lett. 96, 057204 (2006).

${ }^{8}$ S. Rusponi, T. Cren, N. Weiss, M. Epple, P. Buluschek, L. Claude, and H. Brune, Nat. Mater. 2, 546 (2003).

${ }^{9}$ S. Chikazumi, Physics of Ferromagnetism (Oxford University Press, New York, 1997).

${ }^{10}$ D. S. Chuang, C. A. Ballentine, and R. C. O'Handley, Phys. Rev. B 49, 15084 (1994).

${ }^{11}$ R. K. Kawakami, E. J. Escorcia-Aparicio, and Z. Q. Qiu, Phys. Rev. Lett. 77, 2570 (1996)

${ }^{12}$ E. J. Escorcia-Aparicio, H. J. Choi, W. L. Ling, R. K. Kawakami, and Z. Q. Qiu, Phys. Rev. Lett. 81, 2144 (1998).

${ }^{13}$ R. Cheng, K. Y. Guslienko, F. Y. Fradin, J. E. Pearson, H. F. Ding, D. Li, and S. D. Bader, Phys. Rev. B 72, 014409 (2005).

${ }^{14}$ A. Enders, D. Peterka, D. Repetto, N. Lin, A. Dmitriev, and K. Kern, Phys. Rev. Lett. 90, 217203 (2003).

${ }^{15}$ A. Enders, D. Repetto, D. Peterka, and K. Kern, Phys. Rev. B 72, 054446 (2005).

${ }^{16}$ D. Peterka, A. Enders, G. Haas, and K. Kern, Phys. Rev. B 66, 104411 (2002).

${ }^{17}$ D. Sander, W. Pan, S. Ouazi, J. Kirschner, W. Meyer, M. Krause, S. Müller, L. Hammer, and K. Heinz, Phys. Rev. Lett. 93, 247203 (2004).

${ }^{18}$ D. Peterka, A. Enders, G. Haas, and K. Kern, Rev. Sci. Instrum. 74, 2744 (2003).

${ }^{19}$ W. Weiss and M. Ritter, Phys. Rev. B 59, 5201 (1999).

${ }^{20}$ D. I. Jerdev and B. E. Koel, Surf. Sci. 513, L391 (2002).

${ }^{21}$ D. Repetto, J. Honolka, S. Rusponi, H. Brune, A. Enders, and K. Kern, Appl. Phys. A: Mater. Sci. Process. 82, 109 (2006).

${ }^{22}$ T. Y. Lee, S. Sarbach, K. Kuhnke, and K. Kern, cond-mat/ 0504337, Surf. Sci. (to be published).

${ }^{23}$ D. Sander, A. Enders, C. Schmidthals, D. Reuter, and J. Kirschner, J. Magn. Magn. Mater. 177, 1299 (1998).
${ }^{24}$ S. Rusponi, T. Cren, and H. Brune, EPF Lausanne, Switzerland (private communication).

${ }^{25}$ U. Starke (unpublished).

${ }^{26}$ H. Brune, Surf. Sci. Rep. 31, 121 (1998).

${ }^{27}$ J. Dorantes-Dávila and G. P. Pastor, Phys. Rev. Lett. 81, 208 (1998).

${ }^{28}$ Overview on L10 magnetism in Scr. Mater. 53, 381 (2005), and following articles.

${ }^{29}$ I. V. Solovyev, P. H. Dederichs, and I. Mertig, Phys. Rev. B 52, 13419 (1995).

${ }^{30}$ P. Ravindran, A. Kjekshus, H. Fjellvaag, P. James, L. Nordström, B. Johansson, and O. Eriksson, Phys. Rev. B 63, 144409 (2001).

${ }^{31}$ D. Repetto, A. Enders, and K. Kern, J. Magn. Magn. Mater. 300, 479 (2006).

${ }^{32}$ A. Abanov, V. L. Kalatsky, V. L. Pokrovsky, and W. M. Saslow, Phys. Rev. B 51, 1023 (1995).

${ }^{33}$ K. De'Bell, A. MacIsaac, and J. Whitehead, Rev. Mod. Phys. 72, 225 (2000)

${ }^{34}$ R. Allenspach and A. Bischof, Phys. Rev. Lett. 69, 3385 (1992).

${ }^{35}$ P. Ferro, R. Moroni, M. Salvetti, M. Canepa, and L. Mattera, Surf. Sci. 407, 212 (1998).

${ }^{36}$ R. Moroni, F. Bisio, M. Canepa, and L. Mattera, Appl. Surf. Sci. 175-176, 797 (2001).

${ }^{37}$ J. Shen, J. P. Pierce, E. W. Plummer, and J. Kirschner, J. Phys.: Condens. Matter 15, R1 (2003).

${ }^{38}$ L. Neél, J. Phys. Radium 15, 376 (1954).

${ }^{39}$ W. Weber, C. H. Back, A. Bischof, D. Pescia, and R. Allenspach, Nature (London) 374, 788 (1995).

${ }^{40}$ E. J. Escorcia-Aparicio, J. H. Wolfe, H. J. Choi, W. L. Ling, R. K. Kawakami, and Z. Q. Qiu, Phys. Rev. B 59, 11892 (1999).

${ }^{41}$ H. C. Mireles and J. L. Erskine, Phys. Rev. Lett. 87, 037201 (2001).

${ }^{42}$ C. Boeglin, S. Stanescu, J. P. Deville, P. Ohresser, and N. B. Brookes, Phys. Rev. B 66, 014439 (2002).

${ }^{43}$ F. Jona and P. M. Marcus, Crit. Rev. Surf. Chem. 4, 189 (1994).

${ }^{44}$ The epitaxial strain can be calculated to $9.2 \%$ by assuming a lattice constant of fcc-Fe, $a_{\mathrm{Fe}}^{f c c}=3.59 \AA$ (Ref. 43), and of Pt, $a_{\mathrm{Pt}}^{f c c}=3.92$.

${ }^{45}$ We found this number in growth simulations based on the Monte Carlo method without surface diffusion, as is expected at such low growth temperature.

${ }^{46}$ K. Kuhnke and K. Kern, J. Phys.: Condens. Matter 15, S3311 (2003).

${ }^{47}$ M. Komelj, D. Steinauf, and M. Fähnle, Phys. Rev. B 73, 134428 (2006). 